\title{
Proceso de enseńanza-entrenamiento de jóvenes en el fútbol: posibilidades a partir de un modelo ondulatorio
}

\section{Coaching young in soccer: possibilities from a wavy model}

\author{
Otávio Baggiotto Bettega ${ }^{1}$, Larissa Rafaela Galatti ${ }^{1}$, Sérgio José Ibáñez Godoy ${ }^{2}$, \\ Antonio Antunez Medina ${ }^{2}$ y Alcides José Scaglia ${ }^{3}$ \\ 1 Universidade Estadual de Campinas (Brasil) \\ 2 Universidade de Extremadura (España) \\ 3 Universidade Estadual de Campinas (Brasil)
}

\begin{abstract}
Resumen: El proceso de enseñanza-entrenamiento del fútbol debe ser coherente con el contexto en que se realiza, considerando los problemas y potencialidades, en busca de calificar las acciones técnico-tácticas y proporcionar la mejora en el juego. En esta perspectiva, este estudio de naturaleza teórica tiene como objetivo presentar un modelo ondulatorio para la enseńanza-entrenamiento del fútbol. Así, se presenta el modelo ondulatorio para el fútbol, adaptado de Ibáńez (2011), como una manera de ayudar en la organización y sistematización de los contenidos táctico-técnicos de ataque y defensa durante las etapas de formación, así como orientar la aplicación y evaluación de este proceso.

Palabras clave: Fútbol; Modelo ondulatorio; Proceso de enseńanza-entrenamiento.
\end{abstract}

Abstract: The design of a youth athlete development process in soccer must be coherent with the major context, considering challenges and potentialities, and qualifying tactical-technical competences. Considering that, this theoretical study aims to present a "wavy model" for soccer, adapted from Ibáńez (2011), as a supporter for the organization and systematization of the tactical-technical defensive and offensive game phases, as much as insure the coaching and assessment process.

Key words: Soccer, Wavy Model, Tactical-technical.

\section{Introducción}

La enseñanza del fútbol incita al reconocimiento de las relaciones presentes en el juego, por lo que el acercamiento entre el entrenamiento y el juego, y el juego y el entrenamiento se vuelven indispensables para reflexionar en el proceso de formación del futbolista. El contenido del juego siempre tiene un cierto grado de novedad, imprevisibilidad e incertidumbre, pero su organización inicial como componente de entrenamiento, implica un posible escenario que proyecta un diseño anticipado del entrenamiento (Garganta \& Gréhaigne, 1999). De este modo, definir estrategias ofensivas y defensivas para delimitar las acciones táctica-técnicas de los jugadores preestabelece pistas, ayudando a la exploración del escenario complejo que configura el juego del fútbol (Garganta \& Cunha e Silva, 2000).

El fútbol está presente en una gran variedad de lugares de práctica, calles, plazas, escuelas, clubes, entre otros. En el caso de Brasil el contexto informal es actualmente reducido, pero abarca posibilidades de juego para el aprendizaje de los jóvenes (Damo, 2005). En cada ambiente, el juego se diversifica en sus participantes y objetivos, constituyendo un escenario específico. Sin embargo, el juego como proceso su-

Dirección para correspondencia [Correspondence address]: Otávio Baggiotto Bettega. Universidade Estadual de Campinas (Brasil). otavio.b.bettega@gmail.com pone el punto de interacción entre las personas, modulando las relaciones y extrapolando los sentimientos y significados. Scaglia $(2003 ; 2011)$ presentó el fútbol dentro de la familia de los juegos con balón que se ejecutan con los pies, teniendo semejanzas en cuanto a los patrones organizacionales y diferencias en cuento a la diversidad de respuestas, exigencias y emergencias. Es decir, la forma en que se organiza el acto de jugar se refleja en los comportamientos y en los aprendizajes.

En el caso de las categorías de formación de los clubes de fútbol, la periodización del entrenamiento todavía provoca conflictos, sobre todo en el tratamiento de los contenidos táctico-técnicos (Bettega, Scaglia, Morato \& Galatti, 2015; Galatti, Scaglia, Bettega \& Paes, 2016). La complejidad y la aleatoriedad del juego dificultan la transferencia de los contenidos para el contexto de entrenamiento, principalmente cuando se visualizan de forma más positivista en el control de cargas. Es decir, el juego es el punto culminante de la libertad como señala Sérgio (2003), en una perspectiva fractal que transita entre la permanencia y la variabilidad (Cunha e Silva, 1995). Incluso cuando el proceso de aprendizaje esta planificado y dirigido por el entrenador, las interacciones entre los individuos que juegan condicionan sus respuestas motrices, puesto que el aprendizaje no es lineal. Por ello, la variabilidad, la complejidad y la no linealidad del aprendizaje ganan relevancia en el ambiente de entrenamiento. 
En el caso de la periodización de contenidos táctico-técnicos para la enseñanza de los deportes y la formación de los jóvenes, Ibáñez (2008) señala que el entrenador es responsable de seleccionar adecuadamente las actividades que se desarrollarán en cada etapa de la formación, animando a los jóvenes a tomar decisiones en situaciones reales de juego, es decir, el entrenador no debe imponer soluciones a las tareas de entrenamiento, sino facilitar que los jugadores tengan autonomía para resolver los problemas de juego. En general, el proceso de planificación establece un papel entre el currículum de formación, la instrucción del entrenador y el aprendizaje del deportista (Hall \& Smith, 2006).

Defender y atacar implican acciones que apuntan a la superposición al oponente, siendo éstas, condicionadas por un contexto táctico y concretadas por medio de una técnica. La táctica se caracteriza como la red compleja de acontecimientos donde se unen las acciones de los jugadores en torno a sus objetivos ofensivos y defensivos. Es decir, presupone la existencia de una concepción unitaria a través de acciones individuales y colectivas para el desarrollo del juego (Teodorescu, 2003). La técnica consiste en la forma de representación de las intenciones tácticas de los jugadores, una acción motora específica del deporte que busca optimizar las condiciones para la solución de los problemas de juego, con eficiencia y eficacia (Galatti, Bettega, Reverdito, Montero, Paes \& Scaglia, 2017; Garganta, 1997).

Así pues, el objetivo del estudio de este artículo se centra en la presentación de un modelo ondulatorio de enseñanzaentrenamiento para el fútbol, teniendo como base teórica tres modelos teóricos: (a) el Modelo Ondulatorio Progresivo para la Enseñanza/Entrenamiento de los Deportes de Equipo (MOPEDE) de Ibáñez (2011); (b) el Modelo de Desarrollo Deportivo propuesto por Côté, Baker y Abernethy (2007); (c) el Modelo de Organización Especifico para el fútbol propuesto por Bettega y colaboradores (2015) que tiene principios relevantes para el proceso de enseńanza-entrenamiento y que consta de cuatro etapas: iniciación (9 años - 11 años), transición (12 años - 14 años), especialziación (15 años - 17 años) y consolidación ( $\geq 17$ años), Por lo tanto, se presenta el Modelo Ondulatorio No Lineal Aplicado al fútbol, que se fundamen- ta a partir de las teorías interaccionistas y está fundamentado en algunos principios, tales como: una contextualización táctica y técnica, la visión constructivista del aprendizaje, la consideración de las etapas del aprendizaje, estilo de enseñanza que fomenta la resolución de problemas y la existencia de un programa de entrenamiento.

\section{Modelo Ondulatorio Aplicado al Fútbol}

En la alternancia de las fases de ataque y defensa en las diferentes etapas de la formación, el desarrollo de los contenidos contemplará distintos períodos de transición entre las fases del juego, y es a partir de las cuales el MOPEDE prevé la periodización del entrenamiento. La primera preocupación para el entrenador consiste en cómo definir bloques de contenidos táctico-técnicos de entrenamiento, considerando el nivel más bajo de comprensión en las etapas iniciales a partir del desarrollo mental progresivo de un estado de menor equilibrio a un estado de equilibrio superior (Piaget, 2012). Los cambios en la capacidad de la memoria de trabajo, así como en la creciente cantidad de conocimiento almacenado en la memoria a largo plazo durante la transición a la adolescencia tienden a facilitar el procesamiento de diferentes informaciones, así como a la mejora de la capacidad de aprender, recordar y razonar (Papalia \& Feldman, 2013).

El entrenador para hacer frente a la enseñanza del fútbol necesita utilizar procedimientos pedagógicos congruentes con la etapa de formación en la que interviene y con el contexto en el cual está insertado, principalmente en las primeras etapas que contemplan la base del aprendizaje para las siguientes etapas (Cañadas e Ibáñez, 2010). De este modo, la estructura utilizada para el juego, el número de jugadores, la propuesta de reglas, así como la introducción de conceptos de juego, deben respetar el nivel de comprensión de los deportistas, aumentando gradualmente de forma no lineal durante el proceso formativo. Como se expone en la figura 1, esta comprensión de la no linealidad procede de la relación entre los individuos, sus características biológicas particulares, así como de sus contextos sociales de participación, establecidos en medio del contexto deportivo y sus especificidades.

\section{Procedimientos Pedagógicos}

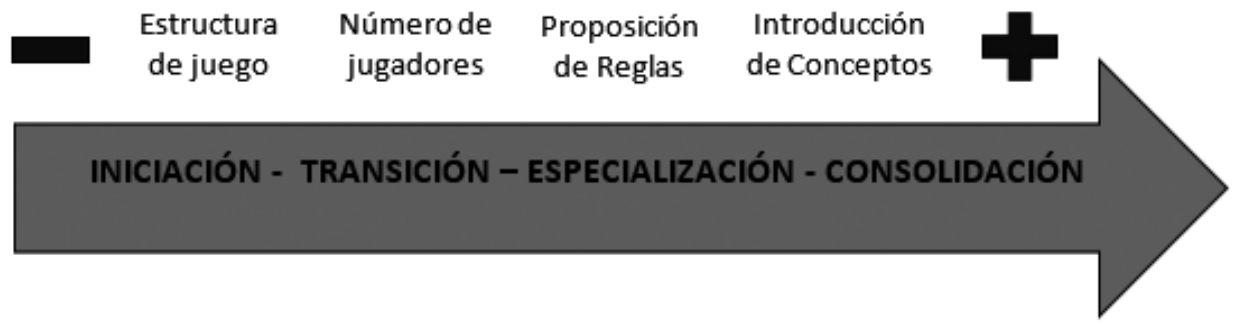

Figura 1. Propuesta de procedimientos pedagógicos durante el proceso formativo. 
La manipulación de los procedimientos pedagógicos es un proceso de toma de decisiones preactivas, que ocurre en parte a priori, visualizado en el plano de la creación y estructurado a partir de las condiciones iniciales, pero también tiene ocurrencia circunstancial, operacionalizado y restablecido sobre la base de las demandas momentáneas dispuestas de la relación con el contexto de interacción, decisiones interactivas. De este modo, el estudio de Araújo y colaboradores (2010) resaltaron que el ambiente de práctica de jóvenes jugadores brasileños y la elevada variabilidad de las limitaciones eran los condicionantes del aprendizaje deportivo, identificando que las interacciones en estos condicionantes no tenían la mediación de un entrenador. Así, se destaca que los ambientes actuales de práctica del fútbol, que generalmente están compuestos por la presencia del entrenador, deben tener presentes estos condicionanates, siendo estos estructurados en el diseño de las tareas de entrenamiento, no con respuestas previamente establecidas o cerradas, sino empleando constante de la presentación de problemas y por consiguiente de la ayuda en la resolución (Corcho, González \& Arrojo, 2017). Es decir, el entrenador actuando como un generador de problemas y también como un mediador del proceso.

A partir del conocimiento del contexto, se propone la periodización de los contenidos de la fase de juego de ataque y defensa a partir de las diferentes etapas de formación (iniciación, transición, especialización y consolidación). El énfasis dado en los contenidos se dividirá en períodos diferentes para cada etapa. Sin embargo, en cada período se propone un enfoque subjetivo de contenidos desarrollados, como se muestra en el ejemplo: $1^{\circ}$ período - 70\% de contenidos ofensivos y 30\% de contenidos defensivos; $2^{\circ}$ período - $30 \%$ de contenidos ofensivos y $70 \%$ de contenidos defensivos. Esta propuesta teórica de modelo ondulatorio deberá ser ajustada por cada entrenador a partir de las características de su equipo. Se destaca que en cada período se desarrollarán más contenidos de una u otra fase del juego y que el porcentaje dependerá de los objetivos del entrenador y de las demandas del contexto. En esta disposición, se presenta el gráfico 1 con la alternancia de los contenidos de ataque y defensa durante los diferentes períodos de la temporada.

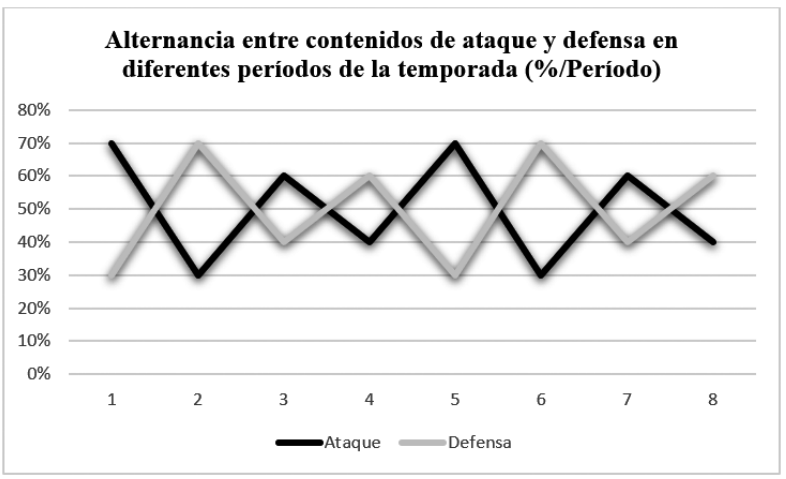

Gráfico 1. Ejemplo de estructuración de la temporada a partir de la alternancia de las fases de juego.
Complementando la organización del gráfico 1 se proponen períodos de transición entre las fases del juego, organizados con mayor duración en las primeras edades, conforme el gráfico 2. Así, en el período de iniciación estaría compuesto por un Mesociclo (treinta días), posibilitando una amplia vivencia de los contenidos seleccionados para ese período. En el período de transición ya se trabajaría con una mayor alternancia de contenido durante medio Mesociclo (quince días); En la etapa de especialización se propone una gran alternancia de contenidos, un Microciclo (siete días), dado que los deportistas ya tienen conocimiento del juego para identificar y responder a estímulos táctico-técnicos propios de diversos momentos del juego. Por último, al deportista adulto, en la llamada fase de consolidación, se recomienda la sistematización del trabajo por sesión de entrenamiento (incluso para atender el calendario con muchos entrenamientos y partidos).

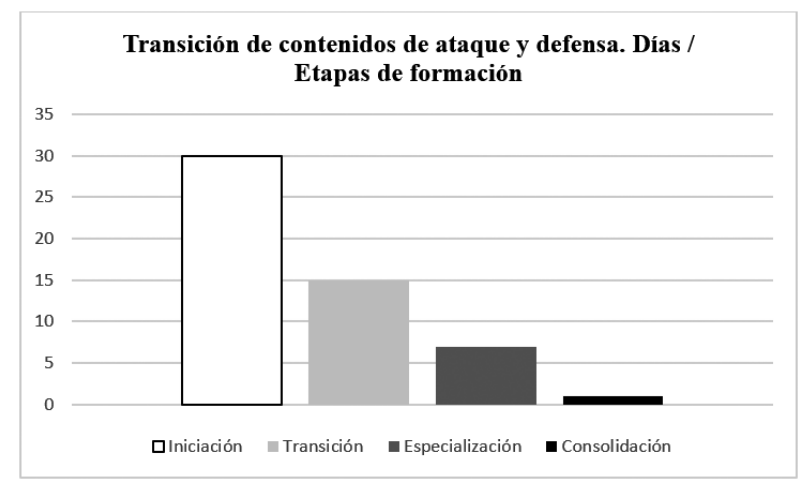

Gráfico 2. Representación de las etapas de formación y duración de los períodos de aplicación de los contenidos seleccionados a partir de la transición entre las fases de juego.

Para justificar esta propuesta desde una perspectiva social, se destaca que la sociedad contemporánea se caracteriza como "sociedad de la información”, pues constantemente se está siendo bombardeado por informaciones y a veces se cree que el conocimiento se establece bajo la forma de información y que el aprendizaje sucede por la adquisición y procesamiento de esa información (Bondía, 2002). Por otra parte, se señala que la velocidad con que se divulgan los acontecimientos y la obsesión por la novedad limitan la experiencia, la relación proximal con las situaciones, y así el aprendizaje significativo de los contenidos. La enseńanza de los contenidos debe establecer oportunidades para que los niños y jóvenes obtengan diferentes experiencias, es decir, que el aprendizaje de los contenidos ocurra por medio de experiencias significativas y que posibiliten profundidad en el aprendizaje. Así pues, resulta relevante organizar el tiempo de desarrollo de los contenidos para las diferentes etapas de formación.

Los nińos y los adultos muestran un alto grado de variabilidad en los niveles entre tarea y contexto, incluso con tareas 
que son lógicamente similares. Las personas poseen diferentes competencias en distintos contextos y estados emocionales, luego varían constantemente sus actividades para adaptarse a condiciones variables, así como reorganizar sus habilidades para hacer frente a nuevas situaciones, personas y problemas. Inconscientemente las personas alteran las relaciones reales entre los sistemas participantes en la percepción, la anticipación, la ejecución motora, la memoria y así sucesivamente (Fischer \& Bidell, 2006). De este modo, el reconocimiento de la etapa de desarrollo de las personas y la aplicación coherente de actividades que concuerdan a esta etapa debe ser pensada como un proceso dinámico, que se vuelve también dependiente de las condiciones circunstanciales de la persona y del contexto.

El cambio de perspectiva de objetos hacia las relaciones, así como de las partes hacia el todo resalta la visión sistémica (Capra \& Luisi, 2014), que en ese caso se emplea en la definición de los contenidos. Así, en la etapa de iniciación, las actividades tienen mayor énfasis en la táctica individual, debido al nivel de comprensión de los individuos que juegan. En la etapa de transición, las actividades con énfasis en la táctica individual y grupal ganan mayor espacio en el entrenamiento. La etapa de especialización ya contempla con más constancia actividades con énfasis en la táctica grupal y colectiva. Durante la etapa de consolidación las actividades se dirigen más a la táctica colectiva, conforme indicado en el gráfico 3. El gráfico ejemplifica esa perspectiva con una representación numérica aproximada y no exacta, como una proyección orientadora de una distribución del volumen de los diferentes componentes tácticos.

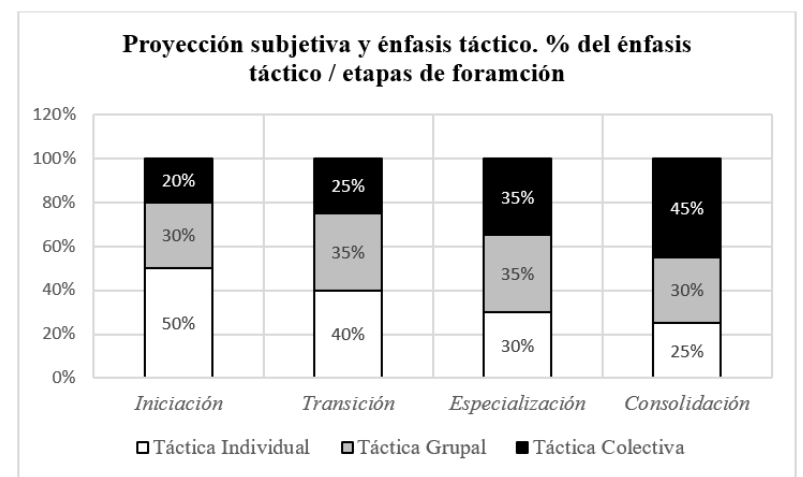

Gráfico 3. Distribución del énfasis táctico abordando las actividades en cada etapa de formación.

Los niños en las edades iniciales perciben de forma limitada a partir de la visualización de "objetos" aislados y al desarrollar sus mecanismos de lenguaje, así como otras funciones comportamentales empiezan a percibir algunas relaciones entre los "objetos" en el ambiente. Esta percepción descontextualizada procede del hecho de que los nińos utilizan la memoria a partir de ejemplos concretos, siendo que el pensamiento abstracto se desarrolla principalmente en la adolescencia, estimulando el pensamiento contextualizado. Así, para los nińos pensar significa recordar y para los adolescentes recordar significa pensar (Vigotski, 2007). De este modo, se sugiere períodos de mayor duración en las primeras etapas como posibilidad de que el contenido sea más enfatizado, debido al grado de entendimiento de los jóvenes, así como con mayor orientación para la táctica individual, utilizando para ello pequeñas estructuras para el juego, también llamadas como small side games (Ejemplo: 1x1, 2x1, 2x2, etc.).

La propuesta de los contenidos de las fases de juego durante la formación de los jóvenes a través del fútbol debe considerar el nivel de comprensión en cada etapa. En general, en la etapa de iniciación el desarrollo ofensivo enfatiza un juego más directo (a través de pases largos), pero presentando opciones para la estructuración de un juego más indirecto (a través de pases cortos). En la defensa se utilizan referenciales basados en el oponente y con poca delimitación espacial. En la etapa de transición, ocurre en el ámbito ofensivo una superposición de un juego indirecto en la relación con un juego directo, principalmente en el sector ofensivo. En la fase defensiva, ocurre el desarrollo de una defensa individual sectorizada con mayor delimitación espacial (Bettega, Scaglia, Morato \& Galatti, 2015).

La etapa de especialización contempla la fase ofensiva a partir de la estructuración de un juego más indirecto, aproximado y con creación de líneas de pase. En lo que se refiere a la fase defensiva se fomenta la defensa individual sectorizada con el desarrollo de referencia zonal. En la etapa de consolidación los contenidos de juego se refuerzan a partir de las características del modelo de juego presentado por el entrenador (Bettega, Scaglia, Morato \& Galatti, 2015). De este modo, se destaca que la planificación de los contenidos en las categorías de base del fútbol necesita ser apoyado por un modelo de enseñanza-entrenamiento y por una propuesta pedagógica desde una perspectiva longitudinal, obteniendo así, la noción de lo que se pretende enseñar, de cómo se anhela enseñar y en qué etapa de formación necesita abordar dicho contenido. Para ello, se presenta la tabla 1 para mostrar de manera general los contenidos que serán desarrollados en cada etapa. 
Tabla 1. Panorama general de los contenidos de defensa y ataque en cada etapa de formación, adaptado de Bettega y colaboradores (2015).

\begin{tabular}{|c|c|c|}
\hline $\begin{array}{c}\text { Fases del juego / } \\
\text { Etapas de formación }\end{array}$ & Defensa & Ataque \\
\hline Iniciación & 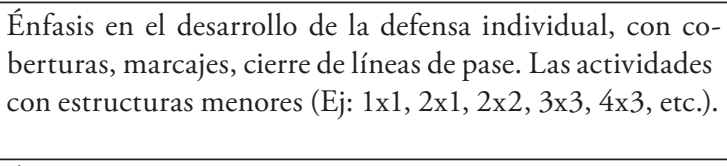 & $\begin{array}{l}\text { Énfasis en el desarrollo de un juego más directo, en pro- } \\
\text { fundidad (através de pases más largos), pero también } \\
\text { con opciones de un juego indirecto (a través de pases } \\
\text { cortos). }\end{array}$ \\
\hline Transición & $\begin{array}{l}\text { Énfasis en el desarrollo de una defensa individual secto- } \\
\text { rizada, con mayor delimitación espacial y referencia po- } \\
\text { sicional. }\end{array}$ & $\begin{array}{l}\text { Énfasis en la superposición de un juego más indirecto } \\
\text { en relación con un juego más directo, principalmente } \\
\text { en el sector ofensivo que ofrece menos riesgos. }\end{array}$ \\
\hline Especialización & $\begin{array}{l}\text { Énfasis en el desarrollo de una defensa zonal con mayor } \\
\text { densidad y con más referencias en cooperación y en la cir- } \\
\text { culación del balón que en el movimiento de los oponentes. }\end{array}$ & $\begin{array}{l}\text { Énfasis en el desarrollo de un juego más indirecto, con } \\
\text { equipo compacto y líneas de pase próximas, realizando } \\
\text { un juego de más movilidad y búsqueda de espacios en } \\
\text { la defensa adversaria. }\end{array}$ \\
\hline Consolidación & $\begin{array}{l}\text { Énfasis en la consolidación de los contenidos desarrollados } \\
\text { en las etapas anteriores (Ej: perfeccionamiento de la defen- } \\
\text { sa zonal) y tendencia a adaptación facilitada al modelo de } \\
\text { juego propuesto por el entrenador. }\end{array}$ & $\begin{array}{l}\text { Énfasis en la consolidación de los contenidos desarro- } \\
\text { llados en las etapas anteriores (Ej: juego de ataque con } \\
\text { mucha movilidad y variaciones posicionales) y tenden- } \\
\text { cia a adaptaciones al modelo de juego propuesto por el } \\
\text { entrenador. }\end{array}$ \\
\hline
\end{tabular}

El aumento de informaciones y referencias en el proceso de enseñanza-entrenamiento del fútbol necesita considerar las etapas de desarrollo de los jóvenes. Generalmente se establece la ampliación de elementos sobre la base del aumento de la complejidad. Sin embargo, la complejidad se caracteriza por el número de elementos de un sistema y el nivel de interacción entre estos elementos (Morin, 2002). En este sentido, la complejidad de una actividad de entrenamiento no se produce solamente en el aumento de elementos formales (reglas, jugadores, espacio, objetivos), sino también en el nivel de interacción que cada individuo tiene con las demandas de esa actividad (elementos funcionales), siendo que cada individuo posee experiencias distintas en relación con la actividad propuesta, luego interactúa de forma diferente con los demás. Esto quiere decir que al intentar simplificar un sistema complejo se está destruyendo, a priori, aquello que se busca percibir, es decir, su inteligibilidad (Garganta, 2001).

Por lo tanto, se reitera que el proceso de formación de jugadores de fútbol puede ocurrir por diferentes caminos, mostrando su complejidad y no linealidad. La búsqueda de recetas exactas no es necesariamente aplicable a cada contexto de práctica. Para ello, la propuesta de un modelo y de algunas directrices puede presentar pistas para la operacionalización del proceso y aproximar aún más teoría y práctica.

\section{Implicaciones para el Modelo Ondulatorio aplicado al Fútbol}

La estructuración de los contenidos de enseñanza-entrenamiento del fútbol necesita ser pensada desde su microestructura, es decir, de la actividad hasta la macroestructura, el currículo de formación. Scaglia (2014) apunta que los mo- mentos de la sesión de entrenamiento figuran como parte de los objetivos establecidos en el currículo, así, se hace necesario la interacción de esas partes en la construcción de todo el proceso de enseñanza-entrenamiento. Además, se resalta que la interacción entre las partes (actividad, sesión de entrenamiento, microciclo, mesociclo, macrociclo) sirve para representar al conjunto (currículo de formación).

El entrenador necesita comprender el plan, el entrenamiento y el juego (competición) de forma conjunta y complementaria, o sea, aproximando las acciones e interconectando los objetivos (Bettega, Scaglia, Nascimento, Ibáñez \& Galatti, 2018). El plan (planificación de la temporada y de cada sesión) se caracteriza como componente de más permeabilidad, pues presenta mayores posibilidades de alteración y manipulación de los elementos. El entrenamiento representa la operacionalización inicial, pudiendo ser modificado y redireccionado, dependiendo de la actuación de los jugadores y, por tanto, el juego (competición) presenta menor permeabilidad debido a sus condiciones formales de estructura y reglas, siendo mucho menores las posibilidades de intervención directa (Bettega, Galatti, Schimtz Filho, Tozetto, Longarela \& Scaglia, 2016).

La planificación del entrenamiento debe considerar las condiciones del juego, lo que, en las categorías de formación, es deseado que venga sustentado por un currículo de formación, teniendo en cuenta el contexto competitivo como una parte complementaria de la organización de la temporada. La construcción de las actividades requiere la creación de un contexto que potencia la aparición de algunas situaciones, siendo éstas, sustentadas en función de un objetivo (Gomes, 2008). Así, la dinámica de la actividad se vuelve dependiente también del comportamiento táctico-técnico de los jugadores y de la intervención realizada por el entrenador. 
La organización y control de los contenidos desarrollados durante la temporada ayuda a la evaluación del proceso y planificación de las acciones posteriores. La planificación también debe abarcar las acciones realizadas durante cada mes (mesociclo), cada semana (microciclo) y cada sesión de entrenamiento, estableciendo estrategias pedagógicas y estructurando las actividades a partir de los objetivos propuestos. De este modo, en la tabla 2 se presenta un modelo de microciclo de entrenamiento (Etapa de Especialización) con la organización de las actividades en cada sesión de entrenamiento y sus objetivos en cuanto a las fases del juego enfatizadas. En ese marco se diseña la semana con un total de 20 actividades, siendo 14 actividades con énfasis en la fase de ataque (70\%) y 6 actividades con énfasis en la fase de defensa (30\%).

Tabla 2. Microciclo con la organziación de las actividades a partir de las fases de juego.

\begin{tabular}{|c|c|c|c|c|c|}
\hline Microciclo 1 & & & & & \\
\hline $\begin{array}{l}\text { Ataque: Desarrollar la } \\
\text { Defensa: Desarrollar } 1\end{array}$ & $\begin{array}{l}n \text { de balc } \\
\text { en bloq }\end{array}$ & $\begin{array}{l}\mathrm{r} \text { ofensivo } \\
\text { mación in }\end{array}$ & $\begin{array}{l}\text { ión para la } \\
\text { ctorial. }\end{array}$ & de pase en & \\
\hline Dias / Actividades & Lunes & Martes & Miercoles & Jueves & Viernes \\
\hline Atividad 1 & Ataque & Ataque & Ataque & Ataque & Defensa \\
\hline Atividad 2 & Ataque & Defensa & Ataque & Ataque & Defensa \\
\hline Atividad 3 & Defensa & Ataque & Ataque & Ataque & Ataque \\
\hline Atividad 4 & Ataque & Ataque & Defensa & Defensa & Ataque \\
\hline
\end{tabular}

La planificación de los contenidos y su aplicación práctica en el entrenamiento se orientan por el currículo de formación, pero puede ser flexibilizado a partir de las demandas inherentes al contexto de aplicación. Las estrategias del entrenador pueden variar en la organización de actividades para los contenidos de la fase de ataque y defensa, sin embargo, se subraya que la utilización del juego tiende a ampliar las posibilidades de relación entre los jugadores y fomentar la resolución de problemas. Así, extrapolando las condiciones estructurales, normativas y funcionales y se convierte en una importante herramienta facilitadora del aprendizaje (Scaglia, Reverdito \& Galatti, 2013).

Otro aspecto importante del proceso formativo en el fútbol es la competición, que generalmente está desvinculada del proceso y se utiliza solamente como fuente de evaluación. La competición debe formar parte del modelo de enseñanza-entrenamiento, de forma equilibrada, diversificando la participación de equipos y jugadores, así como atribuyendo intereses más allá de los resultados (victoria, empate o derrota) (Graça, 2013). Los contenidos enseńados requieren ser aplicados en situaciónes competitivas, debiendo la estructura competitiva propiciar y estimular la aparición de comportamientos desarrollados en el ambiente de entrenamiento.

Basándose en el MOPEDE, la organización de la competición podrá dirigir su estructura, normas y funcionalidad del juego para mostrar contenidos táctico-técnicos defensivos y ofensivos, de forma ondulatoria (Bettega, Scaglia, Morato \& Galatti, 2015; Ibáńez, 2011). Las modificaciones en la estructura competitiva pueden ser fundamentadas a partir de los indicadores de juego: estructuración del espacio, comunicación en la acción y relación con la pelota y sobre la base del modo como ocurren en las diferentes etapas de formación (Garganta, 1994). En este sentido, la tabla 3 apunta las etapas de formación, las posibles caracterizaciones del juego y algunas adaptaciones estructurales para la organización de las competiciones.

Tabla 3. Caracterización de la estructura competitiva en distintas etapas de formación. Adaptado de Casáis, Domínguez \& Lago (2009), y Garganta (1994).

\begin{tabular}{lll}
\hline Etapas & Caracterización del juego & Caracterización de la estrutura competitiva \\
\hline Iniciación & Tendencia de aglutinación alrededor del balón y & Fútbol 5 y Fútbol 7 \\
& exceso de comunicación verbal. Visualización y & - Dimensión del campo: $40 \times 20 \mathrm{~m}-50 \times 30 \mathrm{~m}$ \\
& establecimiento de pocas relaciones por medio del & - Tamańo de la portería: $3 \times 2 \mathrm{~m}-4 \times 2 \mathrm{~m} ;$ \\
balón: Muchas relaciones por medio de la utili- & - Tamaño del balón: $58-60 \mathrm{~cm}$ \\
& zación de la visión central. Dificultad en la visión & - Tiempo de juego: $2 \times 20 \mathrm{~min}-2 \times 25 \mathrm{~min} ;$ \\
& periférica y en la integración cognición-acción. & - Número de jugadores y sustituciones: 5 jugadores (sustitucio- \\
& & nes ilimitadas) - 7 jugadores (sustituciones ilimitadas). \\
\hline
\end{tabular}




\begin{tabular}{|c|c|c|}
\hline Etapas & Caracterización del juego & Caracterización de la estrutura competitiva \\
\hline Transición & $\begin{array}{l}\text { La ocupación de espacio ya no se vuelve muy } \\
\text { dependiente de la posición de la pelota. Además de } \\
\text { la comunicación verbal, la comunicación gestual } \\
\text { también comienza a desarrollarse. Uso de la visión } \\
\text { central y periférica. Adecuación de las soluciones } \\
\text { motoras en función de las diversas alteraciones } \\
\text { morfológicas y funcionales. }\end{array}$ & $\begin{array}{l}\text { Fútbol } 7 \text { y Fútbol } 9 \\
\text { - Dimensión del campo: } 50 \times 30 \mathrm{~m}-70 \times 35 \mathrm{~m} \text {; } \\
\text { - Tamaño de la portería: } 5 \times 2 \mathrm{~m}-6 \times 2 \mathrm{~m} \text {; } \\
\text { - Tamaño del balón: } 62-66 \mathrm{~cm} \text {; } \\
\text { - Tiempo de juego: } 2 \times 30 \mathrm{~min}-2 \times 35 \mathrm{~min} \text {; } \\
\text { - Número de jugadores y sustituciones: } 7 \text { jugadores (sustitucio- } \\
\text { nes ilimitadas) - } 9 \text { jugadores ( } 5 \text { sustituciones). }\end{array}$ \\
\hline Especialización & $\begin{array}{l}\text { Mayor comprensión de las funciones y ocupación } \\
\text { de espacio. Aumento de la comunicación gestual y } \\
\text { disminución de la comunicación verbal. Desa- } \\
\text { rrollo del control propioceptivo y de la técnica } \\
\text { especíica de la modalidad. }\end{array}$ & $\begin{array}{l}\text { Fútbol } 9 \text { y Fútbol } 11 \\
\text { - Dimensión del campo: } 80 \times 45 \mathrm{~m}-105 \times 70 \mathrm{~m} \text {; } \\
\text { - Tamańo de la portería: } 6 \times 2 \mathrm{~m}-7,32 \times 2,44 \mathrm{~m} \text {; } \\
\text { - Tamaño de la pelota: } 68-70 \mathrm{~cm} \text {; } \\
\text { - Tiempo de juego: } 2 \times 40 \text { min - 2x45min; } \\
\text { - Número de jugadores y sustituciones: } 9 \text { jugadores (5 sustitu- } \\
\text { ciones) - } 11 \text { jugadores ( } 3 \text { sustituciones). }\end{array}$ \\
\hline Consolidación & $\begin{array}{l}\text { Mayor comprensión de las estrategias colectivas. } \\
\text { Prevalencia de la comunicación motora. Parti- } \\
\text { cipación funcional diversificada. Optimización } \\
\text { de las capacidades propioceptivas y de la técnica } \\
\text { específica de la modalidad }\end{array}$ & $\begin{array}{l}\text { Fútbol } 11 \\
\text { - Dimensión del campo: } 105 \times 70 ; \\
\text { - Tamaño de la portería: } 7,32 \times 2,44 \mathrm{~m} \text {; } \\
\text { - Tamańo del balón: } 68-70 \mathrm{~cm} \text {; } \\
\text { - Tiempo de juego: } 2 \times 45 \mathrm{~min} \text {; } \\
\text { - Número de jugadores y sustituciones: } 11 \text { (3 sustituciones). }\end{array}$ \\
\hline
\end{tabular}

La competición se convierte en herramienta relevante para atender a los intereses, las capacidades y necesidades de los jóvenes, calificando y ampliando la participación y trayendo efectos positivos en el desarrollo de los individuos y de la modalidad (Cardoso, 2007). La inserción de la competición dentro del proceso formativo en el fútbol no tiene que reproducir las condiciones del fútbol profesional, o sea, la estructura competitiva debe adecuarse a los jóvenes y sus características y que no sean los jóvenes los que se adecuen al modelo formal de competición. En la figura 2 se muestran las experiencias competitivas, internas y externas a partir de dos modelos competitivos, el modelo de competición conceptual y el modelo contextual.

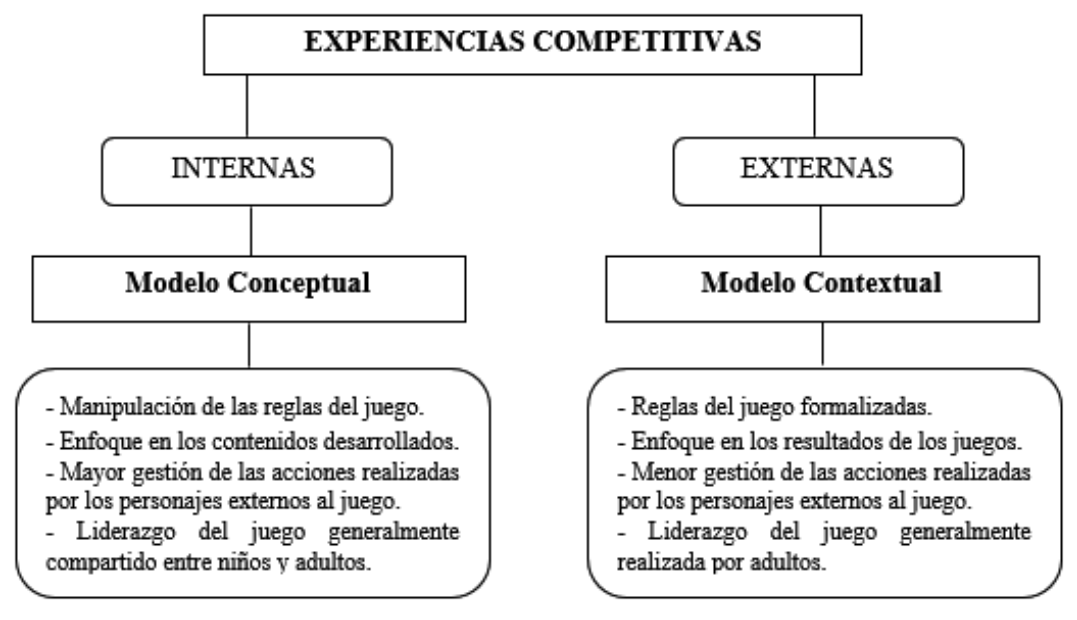

Figura 2. Caracterización de las experiencias competitivas a partir de dos modelos.

El modelo de competición conceptual es una propuesta sustentada por los contenidos de enseńanza, es decir, la construcción de la competición y su énfasis se da a partir de los contenidos desarrollados en determinado momento de la temporada y ocurre internamente al escenario del equipo y del club. Se destaca que este modelo es más apropiado para las etapas iniciales de práctica deportiva, pues tiende a proporcionar un escenario que facilita la socialización, la motivación y el aprendizaje (Strachan, Côté \& Deakin, 2009). El modelo de competición contextual abarca generalmente las condiciones formales de competición establecidas por alguna federación y cuenta con la presencia de otros clubes. 
Por lo tanto, se desea que la formación de los jóvenes jugadores venga apoyada por una planificación a largo plazo, mediada por personas que visualizen jóvenes como jóvenes y no «mini adultos", y que en la aplicación del proceso reconozca los objetivos, las necesidades y predisposiciones de los deportistas, así como las particularidades del contexto en diferentes niveles sistémicos.

\section{Consideraciones Finales}

La resolución de problemas es una demanda constante en el proceso de enseñanza-entrenamiento del fútbol. En ese sentido, se presenta el modelo ondulatorio (MOPEDE) adaptado de Ibáñez (2011) como ayuda a la organización y sistematización de los contenidos en fase de ataque y defensa, así como apoyo a la operacionalización y evaluación del proceso. El modelo se pauta en la alternancia de los contenidos de las fases de juego de ataque y defensa, estableciendo prioridades en cuanto la énfasis en situaciones ofensivas y defensivas durante el transcurso de la temporada.
La propuesta de una actividad de entrenamiento puede ser orientada hacia diversos objetivos, siendo éstos, vinculados a la formación profesional, personal y social del futbolista. Sin embargo, la utilización del modelo ondulatorio busca orientar al entrenador en cuanto a los objetivos dentro de las fases de ataque y defensa, organizando la operacionalización de los contenidos táctico-técnicos y conduciendo las actividades en la misma dirección de las directrices establecidas en la planificación de los contenidos.

Por ello, la utilización del modelo ondulatorio puede ser una importante referencia para la intervención, pero la operacionalización del proceso también depende de las acciones realizadas por el entrenador en la mediación de las interacciones. En esta perspectiva, el entrenador necesita tener competencias para conducir las actividades del entrenamiento sabiendo intervenir a partir de diferentes enfoques, ayudando a los jugadores en la adquisición de nuevos conocimientos, pero también en la oportunidad de autonomía para que los mismos también puedan construir su propio aprendizaje.

\section{Referencias}

1. Araújo, D., Fonseca, C., Davids, K., Garganta, J., Volossovitch, A., Brandão, R. \& Krebs, R. (2010) et al. The role of ecological constraints of expertise development. Talent Development \& Excellence, 2(2), 165-179.

2. Bettega, O.B., Scaglia, A.J., Morato, M. \& Galatti, L. R. (2015). Formação de jogadores de futebol: princípios e pressupostos para composição de uma proposta pedagógica. Movimento, 21(3),791-801.

3. Bettega, O.B., Galatti, L.R., Schmitz Filho, A.G., Tozetto, A. B., Longarela, B. \& Scaglia, A.J. (2016). Planificación táctica en el fútbol: aspectos generales y específicos. E-balonmano: Revista de Ciencias del Deporte, 12(1),45-52.

4. Bondía, J.L. (2002). Notas sobre a experiência e o saber da experiência. Revista Brasileira de Educação, 19, 20-28.

5. Cañadas, M. \& Ibáńez, S.J. (2010). La planificación de los contenidos de entrenamiento de baloncesto en equipos de iniciación. E-balonmano: Revista de Ciencias del Deporte, 6(1),49-65.

6. Capra, F. \& Luisi, P.L. (2014). A visão sistêmica da vida: uma concepção unificada e suas implicaçóes filosóficas, políticas, sociais e econômicas. São Paulo: Editora Cultrix.

7. Cardoso, M. F. (2007). Para uma teoria da competição desportiva para crianças e jovens: um estudo sobre os conteúdos, estruturas e enquadramentos das competiçóes desportivas para os mais jovens em Portugal. Tese (Doutorado no ramo da Ciência do Desporto). Faculdade de Ciências do Desporto e da Educação Física, Universidade do Porto.

8. Casáis, L., Domínguez, E. \& Lago, C. (2009). Fútbol Base: el entrenamiento em categorias de formación. Volume I. Madrid: MCsports.

9. Côté, J., Baker, J. \& Abernethy, B. (2007). Practice and play in the development of sport expertise. In: Eklund, R. \& Tenenbaum, G. (Eds.). Handbook of Sport Psychology. Hoboken: Routledge.

10. Corcho, H.M., González, C.Á., \& Arrojo, A. (2017). Optimización del pensamiento táctico a partir de juegos simplificados de fútbol en iniciación deportiva. SPORT TK-Revista Euroamericana de Ciencias del Deporte, 6, 1, 81-90.

11. Cunha e Silva, P. (1995). O lugar do corpo. Elementos para uma cartografia fractal. (Tese de Doutorado). Faculdade de Ciências do Desporto e da Educação Física, Universidade do Porto.
12. Damo, A. (2005). Do dom a profissão: uma etnografia do futebol de espetáculo a partir da formação de jogadores no Brasil e na França. 2005. $435 \mathrm{f}$. Tese (Doutorado em Antropologia Social)-Instituto de Filosofia e Ciências Humanas, Universidade Federal do Rio Grande do Sul,Porto Alegre.

13. Fischer, K. \& Bidell, T. (2006). Dynamic development of action and thought. En: Lerner, R. (Org.).Handbook of Child Psychology. (pp.313399). Hoboken: Routledge.

14. Galatti, L.R., Scaglia, A.J., Bettega, O.B. \& Paes, R. (2016). Coaches' perceptions of youth players' development in a professional soccer club in Brazil: paradoxes between the game and those who play. Sports Coaching Review, 5(2), 174-185.

15. Galatti, L.R., Bettega, O.B., Reverdito, R., Montero, A., Paes, R. \& Scaglia, A.J. (2017). O ensino dos jogos esportivos coletivos: avanços metodológicos dos aspectos estratégico-tático-técnicos. Revista Pensar à Prática, 20(3), 639-54.

16. Garganta, J. (1994). Para uma teoria dos jogos desportivos colectivos. In: Graça, A. \&Oliveira, J. (Org.). O ensino dos jogos desportivos. Porto: Editora da Universidade do Porto.

17. Garganta, J. (1997). Modelaçâo táctica do jogo de futebol. Estudo da organizaçáo da fase ofensiva em equipes de alto rendimento. Tese de Doutorado. Faculdade de Ciências do Desporto e da Educação Física, Universidade do Porto.

18. Garganta, J. \& Gréhaigne, J. (1999). Abordagem sistêmica do jogo de futebol: moda ou necessidade. Movimento, 10, 40-50.

19. Garganta, J. \& Cunha e Silva, P. (2000). O jogo de futebol: entre o caos e a regra. Revista Horizonte, 16(91), 5-8.

20. Garganta, J. (2001) Futebol e ciência. Ciência e futebol. Efdportes. Revista Digital, 40.

21. Gomes, M. (2008). O desenvolvimento do jogar segundo a periodização táctica. Madrid: MC sports.

22. Graça, A. (2013). Os contextos sociais do ensino e aprendizagem dos jogos desportivos coletivos. In: Nascimento, J.V., Ramos, V. \& Tavares, F. Jogos desportivos: formação e investigação. Florianópolis: Editora Tribo da Ilha. 
23. Hall, T. \& Smith, M. (2006). Teacher planning, instruction and reflection: What we know about teacher cognitive processes. Quest, 58(4), 424-442.

24. Ibáńez, S.J. (2008). La planificación y el control del entrenamiento técnico-táctico en Baloncesto. En: Terrados, N. \& Calleja, J. (Coord.), Fisiología, entrenamiento y medicina del Baloncesto. (pp. 299-313). Barcelona: Paidotribo.

25. Ibáńez, S.J. (2011). Modelo ondulatorio progresivo para la enseńanzaentrenamiento de los deportes de equipo (MOPEDE). Revista Portuguesa de Ciências do Desporto,11(4), 26-27.

26. Morin, E. (2002). O método I: a natureza da natureza. Porto Alegre: Editora Sulina.

27. Papalia, D. \& Feldman, R. (2013). Desenvolvimento bumano. $12^{\circ}$ Edição. Porto Alegre: AMGH.

28. Piaget, J. (2012). Seis estudos de psicologia. 25 Edição.Rio de Janeiro: Forense Universitária.

29. Scaglia, A.J. (2003). O futebol e os jogos/brincadeiras de bola com os pés: todos semelhantes, todos diferentes.150f. (Tese de Doutorado) - Faculdade de Educação Física, Universidade Estadual de Campinas.

30. Scaglia, A.J. (2011). O futebol e as brincadeiras de bola: a família dos jogos de bola com os pés. São Paulo: Phorte Editora.

31. Scaglia, A.J., Reverdito, R. \& Galatti, L. R. (2013). Ambiente de jogo e ambiente de aprendizagem no processo de ensino dos jogos esportivos coletivos: desafios no ensino e na aprendizagem dos jogos esportivos coletivos. In: Nascimento, J.V., Ramos, V. \& Tavares, F. (Org.). Jogos Desportivos: formação e investigação. Florianópolis: Editora Tribo da Ilha.

32. Scaglia, A.J. (2014). Pedagogia do futebol: construindo um currículo de formação para iniciação ao futebol. In: Nista-Piccolo, V. \&Toledo, E. Abordagens pedagógicas do esporte: modalidades convencionais e não convencionais. Campinas: Papirus Editora.

33. Sérgio, M. (2003). Um corte epistemológico: da educação física à motricidade humana. Lisboa: Instituto Piaget.

34. Strachan, L., Côté, J. \& Deakin, J. (2009).An evaluation of personal and contextual factors in competitive youth sport. Journal of Applied Sport Psychology, 3(21), 340-355.

35. Teodorescu, L. (2003). Problemas de teoria e metodologia nos jogos desportivos. Lisboa: Livros Horizontes.

36. Vigotsky, L.S. (2007). A formação social da mente. $7^{\text {a }}$ Edição. São Paulo: Martins Fontes. 